\title{
Simultaneous recording of continuous arterial pressure, heart rate, and ST segment in ambulant patients with stable angina pectoris
}

\author{
A B DAVIES, V BALA SUBRAMANIAN, P M M CASHMAN, E B RAFTERY \\ From the Department of Cardiology and the Divisions of Bioengineering and Clinical Sciences, Northwick Park \\ Hospital and Clinical Research Centre, Harrow, Middlesex
}

SUMMARY Simultaneous and accurate recording of arterial blood pressure and ST segment changes is fraught with technical difficulties. We have developed a new system to enable accurate reproduction of the electrocardiogram and intra-arterial blood pressure, using a transducer/perfusion unit conventionally used to study hypertensive subjects, linked to a frequency modulated tape recorder. Detailed methods of digital analysis have been developed to process the data. This system has been used to study 22 patients with arteriographically proven severe obstructive coronary artery disease who suffered frequent attacks of angina. Control data from quantified dynamic exercise in the laboratory were used for comparison with the effects of normal daily activities outside the hospital and to test the hypothesis that "double product" (heart rate $\times$ systolic blood pressure) is relevant to the onset of angina in such patients. The most important finding was that both angina and asymptomatic episodes of ST segment depression were invariably accompanied by an increase in heart rate, whereas there was considerable variation in blood pressure changes ranging from an increase to a substantial fall. This suggests that heart rate changes are more important in determining ischaemic episodes than blood pressure. Furthermore, the "double product" was not reproducible during repeated episodes of angina and asymptomatic ischaemia and did not apear to have an important role in the pathogenesis of intermittent myocardial ischaemia in this group of patients.

The precise haemodynamic changes associated with spontaneous bouts of myocardial ischaemia are still unclear because of technical difficulties involved in studies of ambulant subjects. Chest discomfort induced by exercise or emotion is thought to be no more than a crude indicator of ischaemia, and it may. be that electrocardiographic ST segment elevation or depression is more sensitive and specific. ${ }^{1-3}$ Most tape systems for ambulatory electrocardiographic recording are deliberately designed with poor low frequency reproduction in order to ensure a stable baseline. It has been shown that ST segment shifts recorded with these direct mode (DM) systems are liable to artefact and cannot be quantified with any confidence. Frequency-modulated (FM) systems, however, are free from these defects, and can be used to quantify ST segment shifts. ${ }^{45}$ We have adapted an FM system ${ }^{\star}$ to record simultaneous electrocardio-

\#Orford Medilog II.

Accepted for publication 8 March 1983 gram and direct arterial blood pressure on separate channels of the same recorder using a well-known and fully validated transducer system, ${ }^{6}$ and we have shown that it can be used to produce accurate records over prolonged periods of time in fully ambulant patients. ${ }^{7}$ The recorder incorporates facilities for precise timing and identification of events. We have used it to study the haemodynamic changes associated with ischaemia in 22 carefully selected ambulant patients with a diagnosis of obstructive coronary artery disease, using dynamic maximal treadmill exercise testing under standardised conditions as the "control" observation.

\section{Patients and methods}

Twenty-two male patients (aged 50 to 74) were selected for study. They all had a history of stable angina of effort for at least three months. All had diagnostic horizontal ST segment depression during maximal (symptom-limited) treadmill exercise testing 
of at least $1 \mathrm{~mm}$ below the resting level measured from the J-point. Chest pain was the event which terminated exercise in all of them. In each patient coronary arteriography, performed to determine their suitability for surgery, had disclosed severe disease (70\% or greater luminal stenosis in either left main, left anterior descending, circumflex, or right coronary artery). All were normotensive, and no significant ST shifts were observed with hyperventilation or postural changes.

No patient took any medication except short-acting nitrates for relief of angina for at least two weeks before the study. All other antianginal therapy was withdrawn under careful supervision before this time without any significant deterioration in symptoms. Ambulatory electrocardiographic tape recording with an FM system for a minimum of 24 hours had failed to show any episodes of significant ST elevation.

The non-dominant brachial artery was cannulated using a modified Seldinger ${ }^{8}$ technique and a $10 \mathrm{~cm}$ long $0.8 \mathrm{~mm}$ internal diameter Teflon cannula (Vygon) was introduced into the artery. The cannula was connected to a perfusion unit incorporating an Akers transducer by an $80 \mathrm{~cm}$ length of polythene tubing which allowed simultaneous perfusion with heparinised saline and pressure wave form recording. The whole system was worn in a pouch placed on the patient's chest, which reduced the degree of discomfort to a minimum while keeping the connecting wires short and ensured a uniform temperature. Two electrocardiographic electrodes were applied to the patient's chest after meticulous preparation of the skin sites. The electrodes were positioned at the manubrium sterni and V5 position to produce a bipolar CM5 lead. 9

After preparation, all patients rested for a period of 20 minutes and then underwent a maximal symptomlimited treadmill exercise test. They were then allowed to return to their normal activities outside the hospital for at least 24 hours, returning only for intermittent transducer calibration and topping up the reservoir. An event marker was used by the patients to identify the onset and ending of episodes of pain.

Informed written consent was obtained from all subjects before the study, and the project was approved by the Hospital Ethical Committee.

\section{RECORDING}

A Medilog Mark II (FM) recording system was specially modified to record blood pressure as well as the electrocardiogram. ${ }^{7}$ Both data channiels were calibrated, the electrocardiographic channel with a series of $1 \mathrm{mV}$ square waves, and the blood pressure channel with $50 \mathrm{mmHg}$ increments (0 to $250 \mathrm{mmHg}$ ) using a mercury manometer of established accuracy.
A minimum of three blood pressure calibrations were performed during each recording period of 24 hours. Commercially available compatible cassette tapes (TDK ADC 120) were used throughout the study.

\section{DATA ANALYSIS}

The cassette tapes were played back at 60 times recording speed using a modified Oxford PB4 replay deck. The electrocardiographic signal was analysed using a Reynolds Pathfinder (Reynolds Medical Ltd), the ST values being continuously plotted by the ST segment trend system. This system has a validated ectopic-rejection facility, which was used throughout the analysis.

The blood pressure data were evaluated in two ways:

(1) A purpose built optimally damped peak follower allowed systolic and diastolic trends to be simultaneously played out with ST segment and heart rate trends on a multichannel recorder (Watanabe Linearcorder $\mathrm{V}$ ).

(2) Digital values of systolic and diastolic pressure were extracted by means of a preprocessor linked to a mini-computer. Heart rate, derived from the pulse interval time, and digital values of ST segment were stored on 8" floppy discs. Specially designed software enabled calculation of the "double product" (heart rate $\times$ systolic pressure) and further data manipulation. This system was programmed to "flag" all unlikely events on the print-out. These could then be examined by virtue of the strict clock timing, and rejected if artefactual. ${ }^{7}$

\section{Results}

Twenty-two patients were studied, producing a total of 583.5 hours of study ( 22 to 47 hours per patient).

Coronary arteriography had shown that 16 had severe stenoses involving all three major coronary arteries. Four had $70 \%$ stenoses of the left anterior descending and circumflex vessels and four patients had $70 \%$ left main stenoses.

In order to ensure a high degree of uniformity, the recordings from two patients were rejected from the analysis because of multiple ventricular ectopic beats. In one other patient only the exercise period could be satisfactorily analysed. Since accuracy was essential if meaningful conclusions were to be drawn from this study, a rigid criterion for transducer drift of $5 \mathrm{mmHg}$ or more in 24 hours was imposed, and this led to five further recordings being excluded. This left a total of 14 technically satisfactory ambulatory tapes for the final analysis.

DYNAMIC EXERCISE (Fig. 1)

Treadmill exercise produced angina and significant 
Dynamic (treadmill) exercise $(n=20)$ $\begin{array}{lll}\text { ST } & \text { Heart Blood } & \text { Double } \\ \text { segment } & \text { rate pressure } & \text { product }\end{array}$

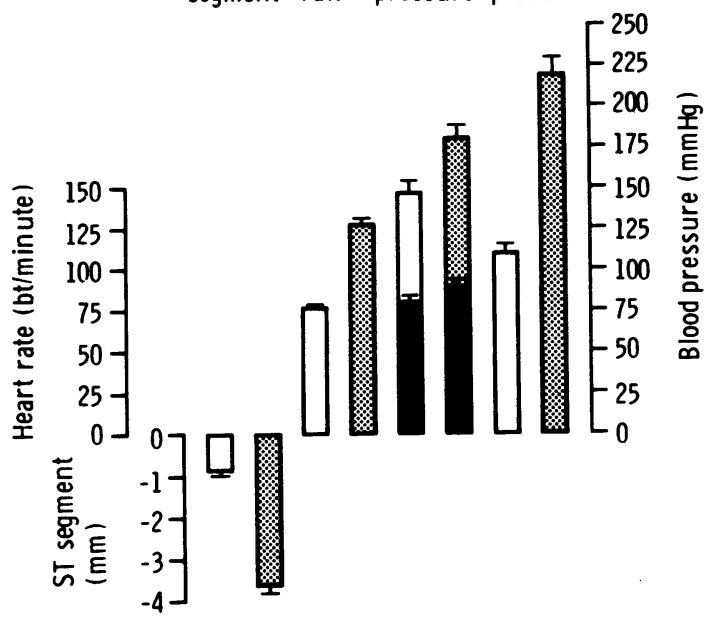

Fig. 1 The changes from resting (open bar) values of ST segment, heart rate, blood pressure, and double product produced by maximal treadmill exercise testing (stippled bar). Diastolic blood pressures blocked in black. All values are mean $\pm S E M$.

ST segment depression in all patients; exercise times ranged from three to 14 minutes. In the 20 patients whose data could be analysed, the ST segment level changed from $-0.9 \pm 0.1$ to $:-3.6 \pm 0.21 \mathrm{~mm}$ :(mean \pm SEM), and the heart rate increased from $78 \pm 1.6$ to $128 \pm 3.4$ beats/minute. There was an increase in systolic pressure from $144 \pm 6.0$ to $179 \pm 6.4 \mathrm{mmHg}$, in diastolic pressure from $80 \pm 2.8$ to $89 \pm 3.4 \mathrm{mmHg}$, and in pulse pressure from $64 \pm 4.3$ to $85 \pm 6.6 \mathrm{mmHg}$. The "double product" rose from $111 \pm 5.4$ to $218 \pm 10.5$ units. All these changes were statistically significant $(p<0.001)$; the directional changes in blood pressure, however, were not consistent within the group, varying from a distinct increase to a fall until cessation of exercise.

\section{AMBULANT PERIOD}

During the period of monitoring 85 episodes of pain were observed in 19 patients. Three patients experienced no pain at all, despite the provocation of angina on the treadmill. After rigorous exclusion of doubtful tapes 56 episodes in 14 patients were available for detailed analysis over a period of 309 hours. The maximum number of episodes of pain experienced by any one patient was 17 .

\section{Episodes of angina}

The maximum values for ST segment depression, heart rate, systolic pressure, and double product occurring in association with pain were "normalised" by expressing them as a percentage of the values recorded during treadmill exercise, where pain was provoked under standardised conditions. Complete accuracy in correlation with event marking and diary recordings could not be guaranteed, so only the maximum values associated with pain were examined.

Pain occurring in the ambulant period was found to be associated with a lesser degree of ST segment depression than during formal treadmill exercise in each patient (Fig. 2A). There was also considerable variation in the degree of ST segment depression associated with spontaneous pain in individuals. Similarly, angina occurring during the ambulant period was associated with lower maximal heart rates than under controlled conditions (Fig. 2B), and there was considerable variability of heart rate within each patient during episodes of pain. Similar results were obtained with maximum systolic pressure (Fig. 2C) and analysis of the maximum "double product" associated with pain showed poor agreement with the control values and wide intra-patient variation (Fig. 2D).

\section{Episodes of significant $S T$ depression}

In order to identify unequivocal episodes of ischaemia from the ST segment changes, "significant" ST segment depression was arbitrarily defined as depression greater than $2 \mathrm{~mm}$ lasting for at least two minutes. These changes were well within the limits of resolution of the recording and playback system. A total of 36 such episodes was observed, 17 of which were accompanied by pain. The greatest number of episodes observed in any one patient was seven. The longest duration was nine minutes, and the maximum ST depression observed was $4.8 \mathrm{~mm}$.

The changes in heart rate and blood pressure accompanying these changes were related to the values observed during the period of supine rest at the beginning of the study in each patient. Significant increases were arbitrarily defined as $10 \mathrm{mmHg}$ for systolic, $5 \mathrm{mmHg}$ for diastolic and pulse pressure, and 20 beats per minute above basal values for heart rate.

Using these arbitrary criteria, it was found that all the recorded episodes of ST segment depression were associated with a significant increase in heart rate. The blood pressure response, however, was not uniform; 31 episodes were associated with a significant increase in systolic pressure and 30 with a significant increase in diastolic pressure while the pulse pressure increased in 33 episodes.

\section{Significant asymptomatic and symptomatic episodes in individual patients}

Five patients had both asymptomatic and painful 
(A)

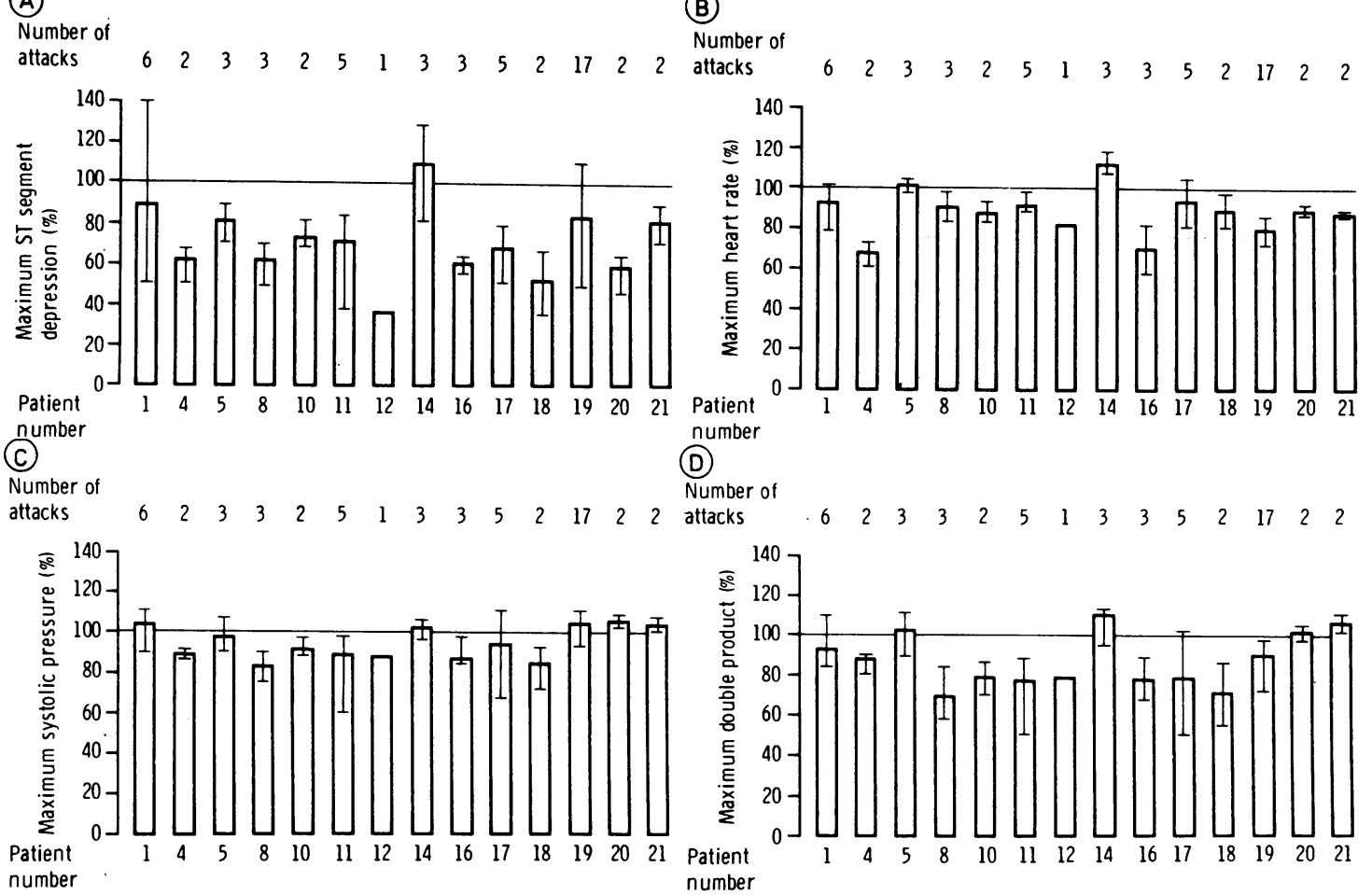

Fig. 2 The mean ( $\pm S E M)$ maximal values of $(A) S T$ segment depression, $(B)$ heart rate, $(C)$ systolic blood pressure, and $(D)$ double product during spontaneous angina in each patient, expressed as a percentage change from those observed during treadmill-induced angina. This illustrates the variability of the response when compared with standardised testing conditions.

episodes of ST segment depression greater than $2 \mathrm{~mm}$ during the period of study. The blood pressure and heart rate changes during these episodes were analysed and compared. The difference in systolic blood pressure between the painful and asymptomatic episodes ranged between 2 and $23 \mathrm{mmHg}$, the mean being $9 \mathrm{mmHg}$. In one patient (case 21) the diastolic pressure was less by $7 \mathrm{mmHg}$ in the painful episodes and in another (case 5) there was no difference. In the other three patients the diastolic pressure changes were between 3 and $16 \mathrm{mmHg}$. The mean difference in double product between painful and asymptomatic episodes was 11 units $(-7 \pm 11)$. No differences in heart rate could be detected.

\section{Discussion}

The mechanism of ST segment changes in the surface electrocardiogram during myocardial ischaemia has not been fully explained. It has been suggested that the changes represent a composite effect of changes in the size and shape of the action potential, secondary to hyperpolarisation of the myocardial cell membrane as a result of ischaemia, ${ }^{10-12}$ but the significance of the direction of the change is not clear. It is thought that depression is an indicator of endocardial ischaemia while elevation only occurs when there is transmural ischaemia. The latter is most likely to occur when there is temporary occlusion of the large epicardial vessels (for example during episodes of coronary spasm). ${ }^{13-15}$ The former is more likely to be seen in situations when myocardial oxygen demand exceeds the possibilities of supply through fixed narrowing of the arteries: the classical mechanistic concept of fixed obstructive coronary artery disease. It must be emphasised, however, that the two situations are not mutually exclusive, and there is evidence to suggest that ST depression not accompanied by increase in heart rate is also the result of spasm, ${ }^{16}$ perhaps of smaller intramural vessels.

The situation is further complicated by the fact that the ST segment is influenced by many factors other than myocardial ischaemia. Changes in posture, hyperventilation, serum electrolyte concentrations, high blood pressure, and certain drugs can also produce ST changes which may be indistinguishable from those related to ischaemia. ${ }^{12}$ The hallmark of myocardial ischaemia is the symptom of angina, but 
many studies have shown that ischaemic ST segment changes may occur without any symptoms, ${ }^{11} 17$ and it is known that spontaneous transient ST segment shifts occur in completely normal subjects. ${ }^{18}$

These considerations indicate the difficulties involved in defining a homogeneous group of patients with a single mechanism of ischaemic ST segment shift. In any study of haemodynamics it is unlikely that all mechanisms will produce the same effects, and conclusions drawn from a heterogeneous group are not likely to be valid. We therefore attempted to define a group of patients who had undoubted, angiographically proven, obstructive coronary artery disease. In all of these patients, angina was closely related to physical exertion, and standardised exercise testing produced ST changes which would be considered diagnostic. There was no evidence of coronary spasm as a major contributing factor in their disease, and all cardiac drugs (apart from nitrates when required) were withdrawn (under careful supervision) for two weeks before the study was performed. Hyperventilation and changes of posture did not produce significant ST changes, all were normotensive, and their serum electrolytes were normal. We considered that this group came as close as possible to the classical mechanistic concept of intermittent myocardial ischaemia.

The technical problems associated with faithful reproduction of ST changes and their interpretation are equally complex. ${ }^{19-21}$ Many of the difficulties can be overcome by using FM recording systems, ${ }^{4}$ which we have shown to satisfy the criteria laid down by Berson and Pipberger. ${ }^{21}$ The analytical system that we used for this study examined the voltage difference between the $P-Q$ isoelectric line and the J point. We recognise that this does not take the ST slope into account as a diagnostic feature, but in all the patients in this study, exercise testing had clearly shown horizontal ST depression at the point of pain.

In order to be as sure as possible that we were only examining ischaemic ST shifts, we imposed strict criteria of depression and duration in defining an "episode" of ischaemia. There can be no doubt that in doing so we have overlooked many episodes of lesser degree, but in view of the uncertainty surrounding the interpretation of ambulatory ST shifts, we felt that this was the only reasonable course to take. Furthermore, the emphasis was placed entirely upon changes; some patients have a consistent ST depression which may lead to uncertainty in interpretation unless it is recognised that this does not necessarily imply continuous ischaemia.

The system that we used to collect the intra-arterial blood pressure data has been fully described and validated, 6 but the use of the FM tape recorder necessitated a complete re-examination. ${ }^{7} \mathrm{We}$ found that the frequency characteristics were much improved by comparison with DM recorders, but that battery depletion was greater. We therefore took great pains to calibrate the system as frequently as possible, and imposed strict criteria for acceptance of the tapes for analysis. A drift of $5 \mathrm{mmHg}$ in 24 hours is not excessive, but since a prime objective of this study was close correlation of accurate data, we felt that all tapes in excess of this value should be excluded. For the same reason, tapes showing excessive arrhythmias were also excluded. The final analysis was performed on only 14 tapes, but we were reasonably sure that any conclusions drawn from these tapes were not open to criticism on the grounds of technical or observational faults.

The most important conclusion from this study is that the mechanistic view of myocardial ischaemia as a simple relation between fixed oxygen supply and variable demand does not appear to hold good, even in this carefully defined group of patients. The concept of "double product" as an indicator of myocardial oxygen consumption ${ }^{22-24}$ is very reasonable, but while it appears to have a close relation to the onset of ischaemia in the experimental laboratory, this would not appear to be true for spontaneous ischaemia. On the contrary, our data suggest that heart rate is a more important and consistent correlate of ischaemia, a view that is supported by the work of other investigators. ${ }^{25}$ The comparison between the findings at the point of pain on controlled exercise and spontaneous bouts of angina also showed that there was no consistent level of ST segment depression at which pain will appear, and the trend charts showed no relation between the duration of ST depression and the experience of pain. These findings lend considerable support to current thoughts on the complexity of the pathogenesis of episodic myocardial ischaemia. ${ }^{26}$

There is evidence that the haemodynamic changes associated with aymptomatic ischaemia occurring in the catheter room differ little from those associated with angina. ${ }^{27}$ This study presented the opportunity to make direct comparisons in the ambulatory situation, but since the indications are that the response to ischaemia is very personal, we felt that it would not be justified to pool data from individuals to describe changes during a "typical" episode. Intra-patient comparisons were more likely to be valid, and five patients had both painful and painless spontaneous episodes during this study. No consistent differences in heart rate, systolic pressure, or diastolic pressure changes could be found. In three patients, a pronounced increase in systolic pressure with painful episodes contrasted with a smaller increase with painless episodes, but the numbers were too small to allow statistical analysis, and more studies must be performed before firm conclusions can be drawn. 
A fall in blood pressure with exercise is considered to be an indicator of left main stem stenosis (or its equivalent) and is thought to indicate severe left ventricular dysfunction during ischaemia..$^{28-30}$ All of our patients had severe disease, and four had significant left main stem lesions, but in only one was there a consistent fall in blood pressure with controlled exercise. There did not appear to be any significant correlation between the severity of the obstructive lesions as judged from the coronary anteriograms and the direction of change of exertional blood pressure.

There has only been one other study of simultaneous blood pressure and ST segment shifts in ambulant patients, ${ }^{31}$ and the reported observations differed from ours. The two studies, however, are not strictly comparable; five of the eight patients studied by Littler et al. were taking beta adrenoreceptor blocking drugs, the DM tape recorder used has since been shown to produce unpredictable ST distortion, ${ }^{4}$ and there was no accurate time coding, so that correlation with symptoms and estimation of heart rates could not have been precise. Nevertheless, these authors found a consistent level of "double product" at the onset of anginal pain, independent of the activity of the patient. This difference may have arisen because the patients studied were less homogeneous than our group; the relatively fixed heart rate produced by the beta adrenoreceptor blocking drugs may have influenced this finding, and we intend to explore this possibility in a further study. These authors also reported that the onset of ST depression coincided with, or came slightly after, the first indication of pain. Our analytical system does not allow any closer temporal correlation than 15 beat averages with any degree of confidence, so we have not so far attempted such correlations, though they would be of considerable interest.

We would like to thank Dr F D Stott and $\mathrm{Mr} \mathrm{H}$ Wolff of the Division of Bioengineering for their constant help and support. Dr V Bala Subramanian is supported by the British Heart Foundation.

\section{References}

1 Master AM, Geller AJ. The extent of completely asymptomatic coronary artery disease. Am $\mathcal{f}$ Cardiol 1969; 23: 173-9.

2 Cohn PF. Silent myocardial ischemia in patients with a defective anginal warning system. Am F Cardiol 1980; 45: 697-702.

3 Maseri A, Mimmo R, Chierchia S, Marchesi C, Pesola A, L'Abbate A. Coronary artery spasm as a cause of acute myocardial ischaemia in man. Chest 1975; 68: 625-33.

4 Bala Subramanian V, Lahiri A, Green HL, Stott FD, Raftery EB. Ambulatory ST segment monitoring: prob- lems, pitfalls, solutions and clinical application. $\mathrm{Br}$ Heart f 1980; 44: 419-25.

5 Krasnow AZ, Bloomfield DK. Artifacts in portable electrocardiographic monitoring. Am Heart $\mathcal{F}$ 1973; 91: 349-57.

6 Millar Craig MW, Hawes D, Whittington J. New system for recording ambulatory blood pressure in man. Med Biol Eng Comput 1978; 16: 727-31.

7 Davies AB, Cashman PMM, Bala Subramanian V, Raftery EB. Simultaneous recording of arterial blood pressure, heart rate and ST segment in the ambulant subject: a new system. Med Biol Eng Comput 1982; (in press).

8 Seldinger SI. Catheter replacement of the needle in percutaneous arteriography; new technique. Acta Radiologica 1953; 39: 368-76.

9 Blackburn H, Katigbak R. What electrocardiographic leads to take after exercise? Am Heart $\mathcal{3}$ 1964; 67: 184-5.

10 Prinzmetal M, Toyoshima H, Ekmekci A, Mizuno Y, Nagaya T. Myocardial ischemia. Nature of ischemic electrocardiographic patterns in the mammalian ventricles as determined by intra-cellular electrographic and metabolic changes. Am f Cardiol 1961; 8: 493-503.

11 Elharrar V, Zipes DP. Cardiac electrophysiologic alterations during myocardial ischemia. Am $\mathcal{F}$ Physiol 1977; 233: H329-45.

12 Surawicz B, Saito S. Exercise testing for detection of myocardial ischemia with abnormal electrocardiograms at rest. Am $\mathcal{F}$ Cardiol 1978; 41: 943-51.

13 Prinzmetal M, Kennamer R, Merliss R, Wada T, Bor N. Angina pectoris 1 . A variant form of angina pectoris. Am f Med 1959; 27: 375-88.

14 Macalpin RN. Correlation of the location of coronary arterial spasm with the lead distribution of ST segment elevation during variant angina. Am Heart $\mathcal{F}$ 1980; 99: 555-64.

15 Maseri A, Severi S, De Nes M, et al. "Variant" angina: one aspect of a continuous spectrum of vasospastic myocardial ischemia. Am f Cardiol 1978; 42: 1019-35.

16 Schang SJ Jr, Pepine CJ. Transient asymptomatic ST segment depression during daily activity. Am $\mathcal{f}$ Cardiol 1977; 39: 396-402.

17 Kunkes SH, Pichard AD, Smith H Jr, Gorlin R, Herman MV, Kupersmith J. Silent ST segment deviations and extent of coronary artery disease. Am Heart $\mathcal{J}$ 1980; 100: 813-20.

18 Armstrong WF, Jordan JW, Morris SN, McHenry PL. Prevalence and magnitude of S-T segment and T- wave abnormalities in normal men during continuous ambulatory electrocardiography. Am $\mathcal{f}$ Cardiol 1982; 49: $1638-42$

19 Hinkle LE Jr, Meyer J, Stevens M, Carver ST. Tape recordings of the ECG of active men. Limitations and advantages of the Holter-Avionics instruments. Circulation 1967; 36: 752-65.

20 Balasubramanian V, Raftery EB, Stott FD. Myocardial ischaemia in patients with frequent angina pectoris [Letter]. $\mathrm{Br}$ Med F 1979; i: 198.

21 Berson AS, Pipberger HV. The low frequency response of electrocardiographs, a frequent source of recording errors. Am Heart $\mathcal{F}$ 1966; 71: 779-89.

22 Robinson BF. Relation of heart rate and systolic blood pressure to the onset of pain in angina pectoris. Circula- 
tion 1967; 35: 1073-83.

23 Gobel FL, Nordstrom LA, Nelson RR, Jorgensen GR, Wang $Y$. The rate pressure product as an index of myocardial oxygen consumption during exercise in patients with angina pectoris. Circulation 1978; 57: 549 56.

24 Amsterdam EA, Hughes JL, DeMaria AN, Zelis R, Mason DT. Indirect assessment of myocardial oxygen consumption in the evaluation of mechanisms and therapy of angina pectoris. Am $\mathcal{F}$ Cardiol 1974; 33: 73743.

25 Wayne EJ, Laplace LB. Observations on angina of effort. Clin Sci 1933; 1: 103-29.

26 Maseri A. Pathogenetic mechanisms of angina pectoris: expanding views. Br Heart $\mathcal{F}$ 1980; 43: 648-60.

27 Chierchia S, Brunelli C, Simonetti I, Lazzari M, Maseri A. Sequence of events in angina at rest: primary reduction in coronary flow. Circulation 1980; 61: 759-68.

28 Thomson PD, Kelemen MH. Hypotension accompanying the onset of exertional angina-a sign of severe com- promise of the left ventricular blood supply. Circulation 1975; 52: 28-32.

29 Levites R, Baker T, Anderson GJ. The significance of hypotension developing during treadmill exercise testing. Am Heart $\mathcal{f}$ 1978; 95: 747-53.

30 Irving JB, Bruce RA, DeRouen TA. Variations in and significance of systolic pressure during maximal exercise (treadmill) testing. Relation to severity of coronary artery disease and cardiac mortality. Am f Cardiol 1977; 39: 841-8.

31 Littler WA, Honour AJ, Sleight P, Stott FD. Direct arterial pressure and the electrocardiogram in unrestricted patients with angina pectoris. Circulation 1973; 48: $125-34$.

Requests for reprints to Dr E B Raftery, Northwick Park Hospital, Watford Road, Harrow, Middlesex HAl 3UJ. 